\title{
ADAPTABLE SOFTWARE STAGE DEVELOPMENT
}

\author{
${ }^{1}$ Todoroi Dumitru, ${ }^{2}$ Todoroi Nicoleta, ${ }^{3}$ Micuşa Dumitru-Victor \\ 1AESM, Chişinău, todoroi@ase.md \\ 2“Gh. Dima” Music Academy, Cluj Napoca, ntodoroi@yahoo.com \\ ${ }^{3}$ ILUM, Chişimău, dimamicusa@gmail.com
}

\begin{abstract}
The exploratory paper investigates Adaptability in general and the Adaptable Tools in particular in quality of Engine to develop Computer Software and Software for Artificial Intelligences. The paper aims to open the discussion around the impact that Adaptable Tools might have on Information, Knowledge Based and Consciousness Societies, Human-Robotic Societies of Information Era. Paper employs an exploratory literature review investigating the past and current state of the art in relation to Adaptability as Engine to create Software for Computers and Artificial Intelligences in Information Era. This literature review serves as the starting point of subsequent theorizing. Based on the literature review we theorize that the Adaptability in general and Adaptable Computing Industry in particular achieves horizon of creation Software for different generations of Computers and Artificial Intelligences with creativity, emotions, temperaments, and sentiments. In this process there are developed new Engine - the Robotic Adaptable Tools (RAT). To name just a few uses of RAT, its can help in: supporting definitions of new robotic intelligence entities, its stratification, and its algorithmic presentation; RAT therefore improve robotic skills and competences; RAT generate requirements for new robotic competences and promote collaborative environment among the Actors of Human - Robotic Society. This paper opens the discussion around succession in creation Software for different generations of Computers and Artificial Intelligences using Adaptable Tools and suggests a wide range of areas for further research in the branch of Robotic Industry. In this paper we argue that by looking at Robotic Adaptable Tools as more than just a set of tools for improving robotic intelligences a Robotic Industry can address some pitfalls of a particular type of Artificial intelligences for Consciousness Society. Adaptable Tools have been developed as part of Software for Computer Industry and they have used in creating Computer Systems for different generations of computers. Robotic Adaptable Tools are a new, but increasingly popular approach, which has been shown to be powerful in many areas of Artificial intelligences creation process. This paper is novel in that it initiates a dialogue around the impact that Robotic Adaptable Tools might have on Robotic Industry.
\end{abstract}

Keywords: Conscience, Artificial intelligence, Creativity, Emotions, Temperaments, Sentiments, Consciousness Society, Human - Robotic Society

\section{Introduction.}

The development and evolution of Microsoft Office and Microsoft Windows shells is based in general on the special methodology of Software creation and implementation such as macros, subroutine, custom commands and specialized features. A major feature of the last decade of Ms Office suite development consists in the ability for users and third-party companies to write addins that extend the capabilities of an application by adding custom commands and specialized features.

Developers Microsoft Office have underlined that in Ms Office 2017 “...the entire user interface has been redesigned to be more intuitive, easier to navigate, and better suited to the task at hand... Now the command you need come to you, depending on the type of object you select and the application you are using". Adaptive Hardware reflects the capability of a system 
to maintain or improve its performance in the context of internal or external changes. Adaptation at hardware levels increases the system capabilities beyond what is possible with software-only solutions. Algorithms, techniques, and their implementation in hardware are developed over a diverse variety of applications. The methodology of the OnOff-line adaptable processors supports development of Adaptable Software. It is composed from the next phase: Definition of new elements (Data or Actions), Processing, Fixation and element's Usage.

It was demoonstrated, that the Adaptable tools as base for creation, application, and development of adaptable software are characterized by a set of advanced linguistic' and processors' features. Adaptable languages, as part of adaptable software and hardware, integrate such linguistics' features as: Multilanguageability, Universality, Speciality, Exensibility, Dialectability, Compactibility of Basis, Reductability, Effectivity of modification, Continuity of Human and Machine experience, Touchability to the Formal Natural Language level of Human-Machine Interactions. Adaptable processors as part of adaptable software and hardware integrate such translation' features as: Universality, Mobility, Transferability, Cognisability, Specializability, Minimizing of Processors' Quantity, and Raising the level of Adaptable Software to the level of Problem Formulation.

1.Adaptable Processors from the Adaptability Cube (Figure no.1) are divided into three levels of translation complexity.

1.1. The first level Adaptable Processors represent a set of adaptable Time-Realization, Model-Realization and Type-Translation type of extensions. The Time-Realization Adaptable Proce-ssors type of extensions is presented by the Adaptable Processors: Preprocessors, Inter-processors and Postprocessors. The Model-Realization Adaptable Proce-ssors type of extensions represents realization using the Level - Level, Level Direct and Level - Level -Direct methods. The third set of first level Adaptable Processors is formed by Adaptable Processors of adaptable Type-Translation type: adaptable
Compilation, adaptable Interpretation and adaptable Compilation-Interpretation of extensions.

The Level - Level type realize extensions by " lowering » method from one level of programming adaptable language (LAP) to another until the base level of programming adaptable language is reached (BLAP) Adaptable Processors of Level -Direct type " lower» extensions-elements from the written program in LAP. Thus they are directly transformed into a program written in BLAP language. Adaptable Processors of Level Level - Direct type are created in the base of processing translation modules of Level Level and Level -Direct type.

1.2. The second level Adaptable Processors presents first level Adaptable Processors combinations of adaptable TimeRealization, Model-Realization and TypeTranslation type of extensions. The second level processors are presented using Adaptable Processors of Time-Model-Realization type, of Type-Model-Realization type and of Type-Time-Realization type of extensions. Theorems of second level of translation complexity Adaptable Processors automatic obtaining of Time-Model-Realization type of extensions are demonstrated in [6] by means of E-T-M Adaptable Processors translation interactions formalisms.

1.3. The third level Adaptable Processors of translation complexity from the Adaptability Cube are represented by means of processors of type Processors-AdaptableCompilers, Adaptable-Processors- Interpreters and Adaptable-Processors-CompilersInterpretation. Adaptable Processors Compilers are presented in [6] using adaptable Preprocessors Compilers, adaptable Interprocessor Compilers and adaptable Postprocessors-Compilers. Theorems of these third levels of translation complexity Adaptable Processors automatic obtaining are demonstrated by means of E-T-M formalisms of Adaptable Processors translation interactions.

\section{E-T-M formalisms of adaptable processing.}

Making the investigation of the Adaptability Cube, one can underline 9 types 
of first level of translation complexity Adaptable Processors, 27 types of second level Adaptable Processors and 27 types of third level Adaptable Processors [6].

There are identified 27 Adaptable Processors schemes. Types of translators are presented by compilation, interpretation and mixed methods: compilation-interpretation. Taking as a base the mode of extensions realization, the processors are grouped into level-level, level-direct and mixed types: level-level-direct. Taking into account the order (time, place) of base extensionselements correlative realization, processors are of preprocessor, inter-processor and postprocessor type. As a result of the research from below there were obtained accumulated Adaptable Processors and demonstrated by the Adaptability Cube.

By means of E-T-M formalism of Adaptable Processors translation interactions, will be demonstrated the affirmations of first level of translation complexity Adaptable Processors automatic creation from Adaptability Cube. E-T-M formalism is also used at demonstration of automatic creation of second level of translation complexity
Adaptable Processors from Adaptability Cube and of third level of translation complexity Adaptable Processors.

\section{First level of translation complexity Adaptable Processors.}

There were used E-T-M formalisms in order to demonstrate translation interactions with first level Adaptable Processors: adaptable processing of Level - Level, Level Direct, and Level - Level -Direct types, adaptable pre-processing, inter-processing, and post-processing, adaptable compilation, interpretation and mixed compilationinterpretation of extensions. These processors forms atomic base of Adaptable Processors from the Adaptability Cube (Figure no. 1) .

The base Adaptable Processors of extensions realization mode are created according with their adaptable level-level (LL), level-direct (L-D), and level-level-direct (L-L-D) processing schemas.

\subsection{Adaptable Processors of level-level} (L-L) type.

General scheme of this processor type is:

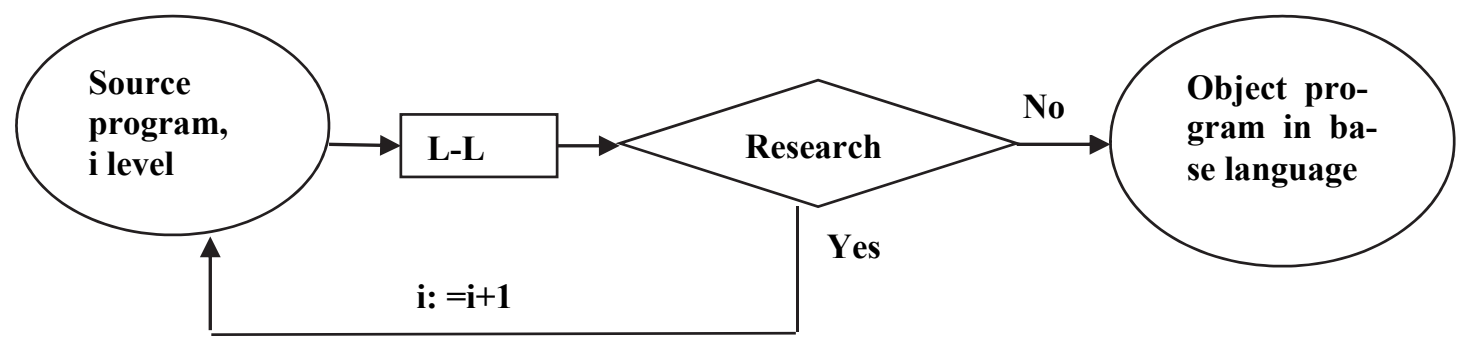

In this scheme the program in adaptable language is presented by „Source program, $\mathrm{i}$ level", processor of level-level type is presented by the processor ,L-L”, „Research” defines if the result of translation still contains extensions (Yes/No) .

As a result, it is obtained „Object program in base language".

Using E-T-M formalism this processor is presented by a row of processors of the following type:

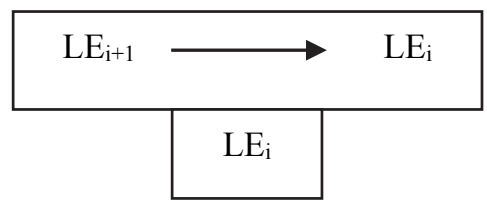

where $\mathrm{i}=0,1,2, \ldots, \mathrm{p}-1$ ( $\mathrm{p}$ - levels number of programming adaptable language) 


\section{The second level Adaptable Processors}

The Adaptable Processors of the second level of translation complexity from the Adaptability Cube represent combinations of the first level Adaptable Processors of Extensions Time-Realization and ModelRealization types and of Adaptable TypeTranslation $[2,6]$. The Processors of the second level are presented by the Adaptable Processors of Time-Model-Realization Extensions type (Table 1.), of Type-ModelRealization Extensions type (Table 2.) and of Type-Time-Realization Extensions type (Table 3.) .

There are demonstrated processes of automatic obtaining of Adaptable Processors of the second level of Time-ModelRealization Extensions type on the basis of ET-M interaction formalism of the Adaptable Processors. The demonstration of automatic obtaining of other Adaptable Processors of the second level will be presented in our following publications.

Pre-processors-L-L, -L-D and -L-L-D form the first set of second level Adaptable Processors. They represent solutions for Adaptable Processors creation, presented by first row of Table1. These pre-processors form one of the next steps in automatic obtaining of Adaptable Processors presented in the Adaptibility Cube.

Inter-processor-L-D Theorem: It is given the base processor and it is necessary to obtain a set of Adaptable Processors of interprocessor-L-D type:

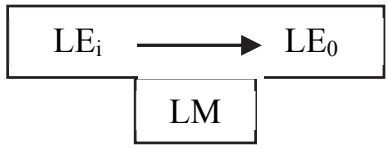

for $\mathrm{i}=0,1,2, \ldots, \mathrm{p}$, that together with (1) form inter-processor-L-D.

For the problem solution is sufficient to create a set (7) of extensions:

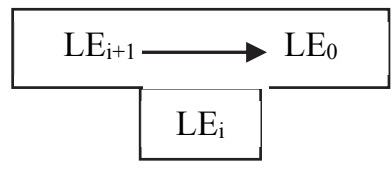

\section{Third level adaptable pre-processors- compilers.}

A third level Adaptable Processor of preprocessor-L-L-compiler type realizes the $f$ program in adaptable programming language LAP, using the order of extensions processing, that are processed using the levellevel processing mode, using compiler's type of processing. As a result, the source program in compilation- pre-processing is the LAP program and LBAP is the object program.

Using Post-processor-L-L-compiler Theorem, Post-processor-L-D-compiler Theorem and Post-processor-L-L-D-compiler Theorem together with Earley formalisms there were automatic obtained the last three Adaptable Processors of the third level from the first plan of Adaptable Processors from the Adaptability Cube (Figure no. 1) .

Thus, was demonstrated automatic obtaining of only one part of Adaptable Processors, and especially of these nine Adaptable Processors of the first level, nine Adaptable Processors of the second level and nine Adaptable Processors of the third level. These processors are universal and specialized.

\section{Adaptable Computing Engine}

It is demonstrated [6] that Adaptable Tools as base for creation, application, and development of Adaptable Software are characterized by a set of advanced linguistic' and processors' features.

Adaptable Languages as part of Adaptable Software integrate such linguistics' features as: Multilanguageability, Universality, Speciality, Exensibility, Dialectability, Compactibility of Basis, Reducability, Effectivity of modification, Continuity of Human and Machine experience, and Touchability to the Formal Natural Language level of Human-Machine Interactions.

Adaptable Processors as part of Adaptable Software integrate such translation' features as: Universality, Mobility, Transferability, Cognisability, Specializability, Minimizing of Processors' Quantity, and Raising the level of Adaptable Software to the level of Problem Formulation. Human social and economic demand and supply for Adaptable Software in the Information and Knowledge Based Societies is too important. 
Table 1. The Adaptable Processors of Time-Model-Realization Extensions type.

\begin{tabular}{|r|c|c|c|}
\hline & Level - Level & Level - Direct & $\begin{array}{c}\text { Level - Level - } \\
\text { Direct }\end{array}$ \\
\hline Pre-processing & \multicolumn{1}{|c|}{ Pre-processor-L- } & Pre-processor-L- & $\begin{array}{c}\text { Pre-processor-L- } \\
\text { L-D }\end{array}$ \\
\hline Inter-processing & \multicolumn{2}{|c|}{ Inter-processor- } \\
L-L & $\begin{array}{c}\text { Inter-processor- } \\
\text { L-D }\end{array}$ & $\begin{array}{c}\text { Inter-processor-L- } \\
\text { L-D }\end{array}$ \\
\hline Post-processing & \multicolumn{2}{|c|}{ Post-processor- } \\
L-L & $\begin{array}{l}\text { Post-processor- } \\
\text { L-D }\end{array}$ & $\begin{array}{l}\text { Post-processor-L- } \\
\text { L-D }\end{array}$ \\
\hline
\end{tabular}

Table 2. The Adaptable Processors of Type-Model-Realization Extensions type.

\begin{tabular}{|c|c|c|c|}
\hline & Level - Level & Level -Direct & $\begin{array}{c}\text { Level - Level - } \\
\text { Direct }\end{array}$ \\
\hline Compilation & Compiler -L-L & Compiler -L-D & Compiler -L-L-D \\
\hline Interpretation & Interpreter-L-L & Interpreter-L-D & Interpreter -L-L-D \\
\hline $\begin{array}{c}\text { Compilation- } \\
\text { interpretation }\end{array}$ & $\begin{array}{c}\text { Interpreter- } \\
\text { Interpreter-L-L }\end{array}$ & $\begin{array}{c}\text { Interpreter- } \\
\text { Interpreter-L-D }\end{array}$ & $\begin{array}{c}\text { Interpreter- } \\
\text { Interpretater-L-L-D }\end{array}$ \\
\hline
\end{tabular}

Table 3. The Adaptable Processors of Type-Time-Realization Extensions type.

\begin{tabular}{|c|c|l|c|}
\hline & Pre-processing & $\begin{array}{c}\text { Inter- } \\
\text { processing }\end{array}$ & Post-processing \\
\hline Compilation & $\begin{array}{c}\text { Compiler-Pre- } \\
\text { processor }\end{array}$ & $\begin{array}{c}\text { Compiler- Inter- } \\
\text { processor }\end{array}$ & $\begin{array}{l}\text { Compiler-Post- } \\
\text { processor }\end{array}$ \\
\hline Interpretation & $\begin{array}{l}\text { Interpreter- Pre- } \\
\text { processor }\end{array}$ & $\begin{array}{l}\text { Interpreter- } \\
\text { Inter-processor }\end{array}$ & $\begin{array}{l}\text { Interpreter - Post- } \\
\text { processor }\end{array}$ \\
\hline $\begin{array}{c}\text { Compilation- } \\
\text { interpretation }\end{array}$ & $\begin{array}{l}\text { Compiler- } \\
\text { Interpreter- } \\
\text { processor }\end{array}$ & $\begin{array}{l}\text { Compiler- } \\
\text { Interpreter- Inter- } \\
\text { processor }\end{array}$ & $\begin{array}{l}\text { Compiler- } \\
\text { Interpreter- Post- } \\
\text { processor }\end{array}$ \\
\hline
\end{tabular}

Adaptable methodology and technology in creation and application of Adaptable Software permit to develop in the future the research process of applicability of each of the first, second, and third levels of Adaptable Processors.

Different types of Adaptable Software will have different domains of its applicability in the process of computerized humanmachine intelligent interaction. This process conducts to develop Natural Language Processing Adaptable Software of humanmachine interaction.

The Adaptable Software forms new industry branch of Informational technologies of the Information and Knowledge Based Societies.

The Ms Office and Ms Windows Systems are developed by Software' shell methodology. Microsoft Office for Mac has for long been criticized. Adaptation at hardware levels increases the system capabilities beyond what is possible with software-only solutions. The methodology of the On-Off-line adaptable processors support development of Adaptable Software and Hardware. It was demonstrated that adaptable tools as base for creation, application, and development of adaptable software are characterized by a set of advanced linguistic' and processors' features.

On the base of adaptable processors of the first level of translation complexity Off-line adaptable processors it is possible to demonstrate the process of automatically creation of the first and second levels of translation complexity On-line and On-Offline adaptable processors.

The demonstrations of automatically creation of On-line and On-Off-line Adaptable Software of the third level of translation complexity can be obtained on the 
base of first and second levels of translation complexity of adaptable processors.

\section{Consciousness Society evolution.}

Sustainable and Healthy societies of our days are the societies which successor is Conscience Society. Based on Social Progress Stage development scientists predicted that the Conscience Society will be created in period from 2019 to 2035 years.

The Future Conscience Society of Information Era as "figure of speech" represents Past Information Society multiplied by the Present Knowledge Based Society. Information Era, except Information and Knowledge based societies, comprises the evolution of society through other intermediate societies such as Internetworking, Creative, and Conscience societies.

7.1. Information Society is based on different active and passive forms of information. The concept of information is related to notions of constraint, communication, control, data, form, instruction, knowledge, meaning, mental stimulus, pattern, perception, and representation

7.2. Knowledge based Society is based on different active and passive forms of scientific knowledge. Science (from the Latin scientia, meaning "knowledge") is, in its broadest sense [4], any systematic knowledge-base or prescriptive practice that is capable of resulting in a prediction or predictable type of outcome. In this sense, science may refer to a highly skilled technique or practice. In its more restricted contemporary sense, science is a system of acquiring knowledge based on scientific method, and to the organized body of knowledge gained through such research.

Science is a continuing effort to discover and increase human knowledge and understanding through disciplined research. Using controlled methods, scientists collect observable evidence of natural or social phenomena, record measurable data relating to the observations, and analyze this information to construct theoretical explanations of how things work. The methods of scientific research include the generation of hypotheses about how phenomena work, and experimentation that tests these hypotheses under controlled conditions. Scientists are also expected to publish their information so other scientists can do similar experiments to double-check their conclusions. The results of this process enable better understanding of past events, and better ability to predict future events of the same kind as those that have been tested.

7.3. Consciousness (Figure no. 1) from the Spiritual point of view is subjective experience, awareness or wakefulness, the executive control system of the mind. It is an umbrella term that may refer to a variety of mental phenomena. Although humans realize what everyday experiences are, consciousness refuses to be defined, philosophers note: "Anything that we are aware of at a given moment forms part of our consciousness, making conscious experience at once the most familiar and most mysterious aspect of our lives."

Consciousness in psychology and philosophy has four characteristics: subjectivity, change, continuity and selectivity. Intentionality or aboutness (that consciousness is about something) has also been suggested by philosopher Franz Brentano. However, within the philosophy of mind there is no consensus on whether intentionality is a requirement for consciousness. Issues of practical concern include how the presence of consciousness can be assessed in severely ill or comatose people; whether non-human consciousness exists and if so how it can be measured; at what point in fetal development consciousness begins; and whether computers can achieve a conscious state.

Some philosophers divide consciousness into phenomenal consciousness, which is experience itself, and access consciousness, which is the processing of the things in experience [6]. Phenomenal consciousness is the state of being conscious, such as when they say "I am conscious." Access consciousness is being conscious of something in relation to abstract concepts, such as when one says "I am conscious of these words.". 


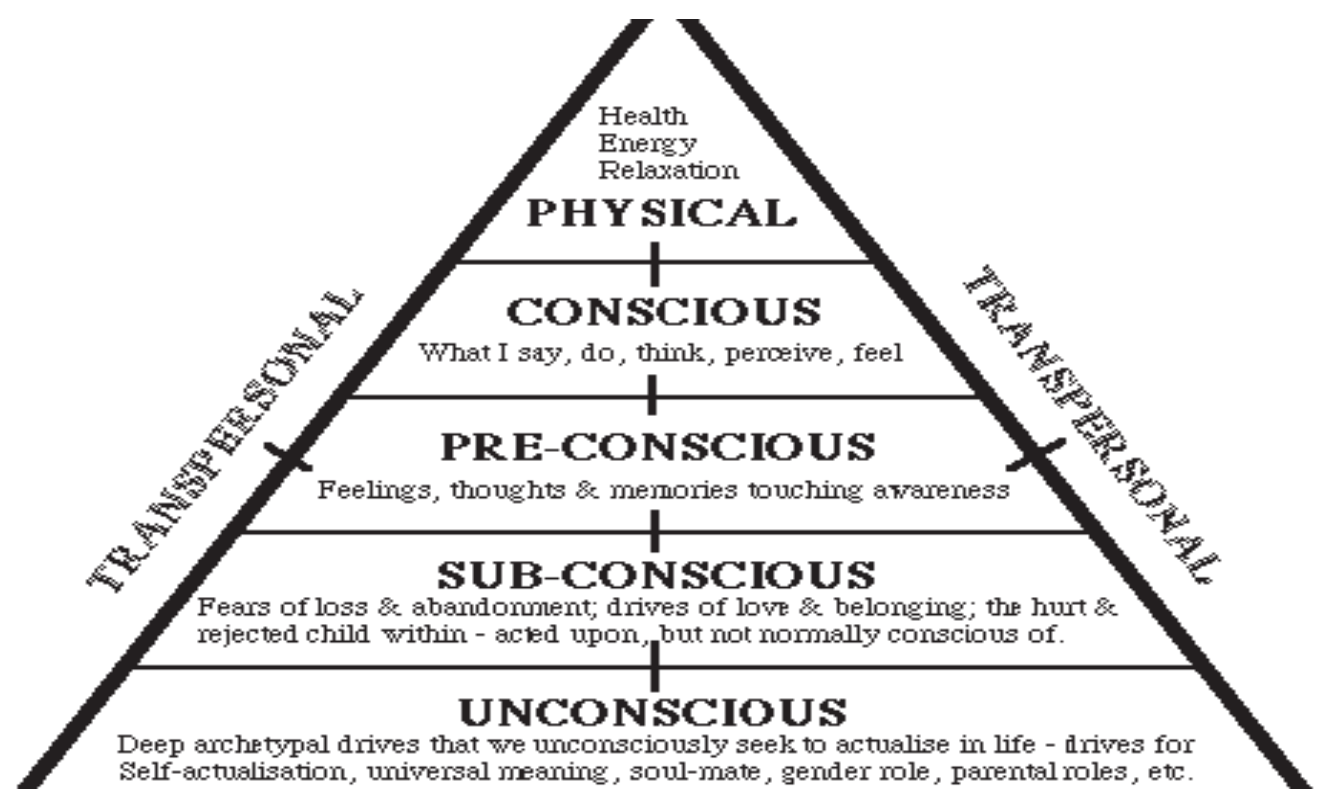

Figure no. 1. Consciousness: subjective experience, awareness, wakefulness, the executive control system of the mind

Various forms of access consciousness include awareness, self-awareness, conscience, stream of consciousness, Husserl's phenomenology, and intentionality. The concept of phenomenal consciousness, in modern history, according to some, is closely related to the concept of qualia (subjective conscious experiences as 'raw feels')

Social psychology links sociology with psychology in their shared study of the nature and causes of human social interaction, with an emphasis on how people think towards each other and how they relate to each other. The behavior and mental processes, both human and non-human, can be described through animal cognition, ethology (scientific study of animal behavior, and a sub-topic of zoology), evolutionary psychology, and comparative psychology as well. Human ecology is an academic discipline that investigates how humans and human societies interact with both their natural environment and the human social environment.

7.4. Consciousness Society represents the higher level of Information Era's societies based materially and spiritually on different active and passive forms of conscience. Conscience from the material and spiritual point of view is ability or a faculty that distinguishes whether one's actions are right or wrong. It can lead to feelings of remorse when a human does things that go against his/her moral values, and to feelings of rectitude or integrity when actions conform to such norms. The extent to which conscience informs moral judgment before an action and whether such moral judgments are, or should be, based wholly in reason has occasioned debate through much of the history of Western philosophy. Commonly used metaphors for conscience include the "voice within" and the "inner light".

\section{Conclusion.}

Present research results evaluate from the Fulbright research project No. 22131 “Societal Information Systems' Adaptable Tools" performed in the University of Omaha at Nebraska, USA [9]. Research results in the AESM institutional Project "Consciousness Society Creation" are announced in tens other 
publications, inclusively in two books $[2,6,8]$.

Detailed information about discussed subject is presented in the Book: "Creativity in Conscience Society. Creativity and Adaptability" [8], which was edited by LAMBERT Academic Publishing, Saarbrucken, Germany in 2012 with next structured presentation: Consciousness evolution; Creativity in Conscience Society; Adaptable Support for Creativity; Adaptable Human - Machine Interface; Creativity's Kernel Extensions-Dialects; Education and research in Conscience Society.

Next book "Creative Robotic Intelligences" [2] is published by Editions Universitaires Europeennes, Saarbrucken, New York in 2017 with next structured presentation: Conscience Society; Creativity is a mental process; Intelligences in Conscience Society; Dedication and Professionalism.

When Creative Dedication and Professionalism at Creativity tops are working, the individuals, the team, the company, and the Society hit success!

Our publication confirms the European Community interest [11] for our research in the Branch of Consciousness Society Creation and its Adaptable Tools - the Software support engine for ROBO-intelligences. The Committee on the Civil problems of the European Parliament endorsed the draft recommendations, as well as the administrative regulations on the civilengineering production of robots. For that document voted PRO: 17 deputies, against: 2 deputies, and Obtained: 2 deputies.

According to data of the European Parliament, in the period 2010-2014 the average sales of robots was $17 \%$ annual and in 2015 has risen to 29 percent. Growth of robots developed the volume of patents in relation to robots - in the last 10 years the volume has doubled.

Artificial intelligence will determine economic efficiency in such spheres as manufacturing, commerce, transport, medical service, education, case-law and agriculture.

It is not yet determined the legal status of robots, which soon will overwhelm us. Scientists are, as some carriers of artificial intelligence, provided with self-education capacity, separately, will need to be identified as "electronic faces" with corresponding Passport.

The document will contain the framework conditions for producers and users of robots, formulated since the great writer Isaac Azimov: 3 principles - the basic conditions in collaboration with robots and humans Asimov's Three Laws of Robotics, as they are called, have survived to the present: (1) Robots must never harm human beings or, through inaction, allow a human being to come to harm; (2) Robots must follow instructions from humans without violating rule 1; (3) Robots must protect themselves without violating the other rules.

\section{References:}

[1] Todoroi, D., Consciousness Society Creation, $6^{\text {th }}$ Edition, April 21-22, 2017, 236 pages, ARA Publisher, University of California Davis, USA, ISBN: 978-1-93592421-0,

http:/www.AmericanRomanianAcademy.org [2] Todoroi, D., Creative Robotic Intelligences, Editions Universitaires Europeennes, Saarbrucken, New York, 2017, 123 pages. ISBN: 978-3-8484-2335-9

[4] Moraru, M., Todoroi D., “About implementation of Pandora gifts in ROBOintelligences", Society Consciousness Computers, Volume 3, May 2016, Alma Mater Publishing House, Bacău, pp. 78-87, (MaterialeleTeleconferinţei Internaţionale a tinerilor cercetători "Crearea Societăţii Conştiinţei”, Ed. a 3-a, 22-23 aprilie 2016) . ISSN 2359-7321, ISSN-L 2359-7321

[5] Guzun, E., Chuciuc, N., Todoroi, N., Todoroi, D., Education process of Emotional ROBO-intelligences, Society Consciousness Computers, Volume 2, March 20-22, 2015, Alma Mater Publishing House, 
Bacău, pp. 50-59, ISSN 2359-7321, ISSN-L Saarbrucken, Germany, 2012, 120 pages. 2359-7321

ISBN: 978-3-8484-2335-4

[6] Todoroi, D., Micuşa, D., Sisteme

[9] Todoroi, D., Nazem, S., Jucan, T., adaptabile, Editura Alma Mater, Bacău, Micusha, D., Transition To A Full România, 2014, 148 pagini. ISBN 978-606527-347-4

[7] Todoroi, D., Consciousness Society Creation, $1^{\text {st }}$ Edition, Materialele Teleconferinţe Internaţionale a tinerilor cercetători "Crearea Societăţii Conştiinţei”, 78 aprilie 2012, Chişinău, 169 pages. ISBN 978-9975-75-611-2.

[8] Todoroi, D., Creativity in Conscience Society, LAMBERT Academic Publishing, Information Society: Stage Development, Working Paper No. 98-2, UNO, Omaha, USA, March 1998. - 38 p.

[10] Todoroi, D., Computer Science. The Adaptable Programming. The Basic Conceptions. ASEM Press, Chisinau, 1992, 76 pages.

[11] Moldova Suverana, 25.01.2017, $\mathrm{Nr}$. 8(2095), utro.ru 